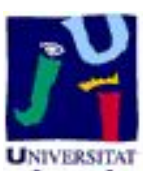

Título artículo / Títol article: Extrinsic isoperimetry and compactification of minimal surfaces in Euclidean and hyperbolic spaces

Autores / Autors

Gimeno García, Vicent ; Palmer Andreu, Vicente

Revista:

Israel Journal of Mathematics, 2013, 194, 2

Versión / Versió:

Preprint de l'autor

Cita bibliográfica / Cita

GIMENO, Vicent; PALMER, Vicente. Extrinsic bibliogràfica (ISO 690): isoperimetry and compactification of minimal surfaces in Euclidean and hyperbolic spaces. Israel Journal of Mathematics, 2013, 194.2: 539553.

url Repositori UJI:

http://hdl.handle.net/10234/85850 


\title{
EXTRINSIC ISOPERIMETRY AND COMPACTIFICATION OF MINIMAL SURFACES IN EUCLIDEAN AND HYPERBOLIC SPACES
}

\author{
VICENT GIMENO\# AND VICENTE PALMER*
}

\begin{abstract}
We study the topology of (properly) immersed complete minimal surfaces $P^{2}$ in Hyperbolic and Euclidean spaces which have finite total extrinsic curvature $\int_{P}\left\|B^{P}\right\|^{2} d \sigma<\infty$, using some isoperimetric inequalities satisfied by the extrinsic balls in these surfaces, (see [22]). Based on estimates on the curvature decay of complete minimal surfaces with finite total extrinsic curvature in Euclidean and Hyperbolic spaces proved by Anderson and De Oliveira in [1] and [21] respectively, we give an alternative proof to the fact that these surfaces are diffeomorphic to a compact surface punctured at a finite number of points. Using this last result and the isoperimetric analysis above alluded, we present a unified proof of the Chern-Osserman inequality satisfied by these minimal surfaces. Finally, we show a Chern-Osserman type equality attained by complete minimal surfaces in the Hyperbolic space with finite total extrinsic curvature.
\end{abstract}

\section{INTRODUCTION}

In the paper [11], A. Huber proved that, when $P$ is a complete, connected and oriented surface with finite total curvature then $P$ is homeomorphic, (in fact, conformally equivalent), to a compact surface punctured at a finite number of points $\bar{P} \backslash\left\{p_{1}, \ldots, p_{n}\right\}$.

On the other hand, S.S. Chern and R. Osserman proved in [4] (using basically techniques from complex analysis) the following estimate for the Euler characterisitic $\chi(P)$ of complete and minimal immersed surfaces $P^{2} \subseteq \mathbb{R}^{n}$, (cmi for short), with finite total curvature, in terms of its total curvature and its number of ends, $k$. This formula is nowadays known as the Chern-Osserman Inequality, and can be stated as

$$
-\chi(P) \leq-\frac{1}{2 \pi} \int_{P} K d \sigma-k
$$

being $K$ the Gauss curvature of $P$.

This inequality is in fact an equality for cmi surfaces $P^{2} \subseteq \mathbb{R}^{n}$ of finite total scalar curvature when we replace $k$ by the (finite) supremum of the volume growth function, (see [1]), so we have

$$
\begin{aligned}
-\chi(P) & =\frac{1}{4 \pi} \int_{P}\left\|B^{P}\right\|^{2} d \sigma-\operatorname{Sup}_{r} \frac{\operatorname{Vol}\left(P^{2} \cap B_{r}^{0, n}\right)}{\operatorname{Vol}\left(B_{r}^{0,2}\right)} \\
& \leq \frac{1}{4 \pi} \int_{P}\left\|B^{P}\right\|^{2} d \sigma-k(P)
\end{aligned}
$$

and therefore, in this case we get a better estimate of the Euler characteristic of the surface using the volume growth of the extrinsic domains $D_{r}=P^{2} \cap B_{r}^{0, n}$ where $B_{r}^{b, n}$ denotes the geodesic $r$-ball in the simply connected real space form $\mathbb{K}^{n}(b)$, (see [7]). These domains $D_{r}$ are known as the extrinsic balls.

When we deal with the same question but considering complete (non-compact) minimal surfaces $P^{2}$ immersed in the hyperbolic space $\mathbb{H}^{n}(b)$, the first consideration is that,

2010 Mathematics Subject Classification. Primary 53A, 53C.

Key words and phrases. Area growth, minimal surfaces, Chern-Osserman inequality, finite topological type, compactification, Euler characteristic.

\# Supported by the Fundació Caixa Castelló-Bancaixa Grants P1.1B2006-34 and P1.1B2009-14

* Supported by MICINN grant No. MTM2010-21206-C02-02. 
by the Gauss equation, the total Gaussian curvature of such surfaces is infinite. However, it is possible to consider surfaces $P^{2} \subseteq \mathbb{H}^{n}(b)$ with finite total extrinsic curvature $\int_{P}\left\|B^{P}\right\|^{2} d \sigma<\infty$. Note that to have finite total scalar (extrinsic) curvature is equivalent to the finiteness of the total Gaussian curvature, when the surface is minimal and immersed in $\mathbb{R}^{n}$.

In view of these considerations, it is natural to wonder if it is possible to stablish a Chern-Osserman inequality for complete minimal surfaces with finite total extrinsic curvature (properly) immersed in the hyperbolic space. This question has been addressed by Q. Chen and Y. Cheng in the papers [5] and [6]. They proved, for a complete minimal surface $P^{2}$ (properly) immersed in $\mathbb{H}^{n}(b)$ and such that $\int_{P}\left\|B^{P}\right\| d \sigma<\infty$, the following version of the Chern-Osserman Inequality, in terms of the volume growth of the extrinsic balls:

$$
\begin{aligned}
& \operatorname{Sup}_{r} \frac{\operatorname{Vol}\left(P^{2} \cap B_{r}^{-1, n}\right)}{\operatorname{Vol}\left(B_{r}^{-1,2}\right)}<\infty \text { and } \\
& -\chi(P) \leq \frac{1}{4 \pi} \int_{P}\left\|B^{P}\right\|^{2} d \sigma-\operatorname{Sup}_{r} \frac{\operatorname{Vol}\left(P^{2} \cap B_{r}^{-1, n}\right)}{\operatorname{Vol}\left(B_{r}^{-1,2}\right)}
\end{aligned}
$$

The proofs of these authors encompasses elaborated computations which depends heavily on the properties of the hyperbolic functions, far from the complex analysis techniques above alluded, used in the Euclidean case.

On the other hand and following in the footsteps of Anderson's unpublished paper [1], G. De Oliveira adressed in the paper [21] the objective to get an estimate for the curvature decay when $P$ is a complete minimal surface in $\mathbb{H}^{n}(b)$ with finite total extrinsic curvature. Although this estimate was not given explicitly, (a fact achieved in the Euclidean context in [1]), it was proved that $\left\|B^{P}\right\|(p)$ goes to 0 as the extrinsic distance $r(p)$ to a fixed point goes to infinity, and then, as in Anderson's paper, it is concluded the properness of the immersion and that the extrinsic distance to a fixed point defined in the submanifold $P, r$, has no critical points outside a compact in $P$. Hence it is possible to construct a diffeomorphism among $P$ and a compact surface punctured at a finite number of points.

We present in this paper a unified approach which encompasses the compactification problem and the proof of one version of the Chern-Osserman inequality (in terms of the volume growth) for complete minimal surfaces with finite total extrinsic curvature immersed in Euclidean or Hyperbolic spaces. We have proved in Theorem 3.1 the following Chern-Osserman inequality, which encompasses inequalities (1.1) and (1.3):

Theorem A. (Theorem 3.1) Let $P^{2}$ be an complete minimal surface immersed in a simply connected real space form with constant sectional curvature $b \leq 0, \mathbb{K}^{n}(b)$. Let us suppose that $\int_{P}\left\|B^{P}\right\|^{2} d \sigma<\infty$. Then

(1) P has finite topological type.

(2) $\operatorname{Sup}_{t>0}\left(\frac{\operatorname{Vol}\left(D_{t}\right)}{\operatorname{Vol}\left(B_{t}^{b, 2}\right)}\right)<\infty$

(3) $-\chi(P) \leq \frac{\int_{P}\left\|B^{P}\right\|^{2}}{4 \pi}-\operatorname{Sup}_{t>0} \frac{\operatorname{Vol}\left(D_{t}\right)}{\operatorname{Vol}\left(B_{t}^{b, 2}\right)}$

where $\chi(P)$ is the Euler characteristic of $P$.

Although with this approach we are not able to state equality (1.2) in the Euclidean setting, we shall prove in Theorem 4.1 the following Chern-Osserman type equality for cmi surfaces in the Hyperbolic space:

Theorem B. (Theorem 4.1) Let $P^{2}$ be a complete immersed minimal surface in $\mathbb{H}^{n}(b)$. Let us suppose that $\int_{P}\left\|B^{P}\right\|^{2} d \sigma<\infty$. Then

$$
-\chi(P)=\frac{1}{4 \pi} \int_{P}\left\|B^{P}\right\|^{2} d \sigma-\operatorname{Sup}_{t>0} \frac{\operatorname{Vol}\left(D_{t}\right)}{\operatorname{Vol}\left(B_{t}^{b, 2}\right)}-\frac{1}{2 \pi} G_{b}(P)
$$


where $G_{b}(P)$ is a nonnegative and finite quantity which do not depends on the exhaustion by extrinsic balls $\left\{D_{t}\right\}_{t>0}$ of $P$ and is given by

$$
\begin{aligned}
G_{b}(P) & :=\lim _{t \rightarrow \infty}\left(h_{b}(t) \operatorname{Vol}\left(B_{t}^{b, 2}\right)\left(\frac{\left(\operatorname{Vol}\left(D_{t}\right)\right)}{\operatorname{Vol}\left(B_{t}^{b, 2}\right)}\right)^{\prime}\right. \\
& \left.+\int_{\partial D_{t}}<B^{P}(e, e), \frac{\nabla^{\perp} r}{\left\|\nabla^{P} r\right\|}>d \sigma_{t}\right)
\end{aligned}
$$

Our unified approach is based on the divergence Theorem and the Hessian and Laplacian comparison theory of restricted distance function, (see [10], [19] and [12]) which involves bounds on the mean curvature of the submanifold. We use in this paper a version of this result for complete minimal submanifolds of real space forms, (see Theorem 2.2), but it can be found more general statements of this theorem, which encompasses complete submanifolds not necessarily minimal in ambient spaces with sectional curvatures bounded from above or from below (see [19] and [12]).

Using these more general results and an extrinsic version of the classical Huber's result, it should be possible to obtain Chern-Osserman inequalities for complete and non-minimal surfaces with finite total extrinsic curvature and properly immersed in Cartan-Hadamard manifolds which displays an appropriate behavior of their sectional curvatures, as it is being studied in [8] and [9], in the line of the results of B. White in [27], where it was presented a version of Chern-Osserman inequality for complete and non-minimal surfaces immersed in $\mathbb{R}^{n}$ with finite total curvature. Other purely intrinsic approach to this question was given by K. Shiohama in [26].

1.1. Outline. The oultline of the paper is following. In Section $\S .2$ we present the basic facts about the Hessian comparison theory of restricted distance function we are going to use (see Theorem 2.2 and Proposition 2.3) obtaining as a corollary the compactification of cmi surfaces in $\mathbb{K}^{n}(b)$ with finite total extrinsic curvature, (Corollary 2.4) and an inequality satisfied by the Euler characteristic of the extrinsic balls in a cmi surface in $\mathbb{K}^{n}(b)$, (Corollary 2.5]. Section $\S .3$ is devoted to the unified proof of the Chern-Osserman inequality for complete minimal surfaces with finite total extrinsic curvature immersed in Euclidean and Hyperbolic spaces (Theorem 3.1), and in Section $\S .4$ it is proved a Chern-Osserman type equality satisfied by the cmi surfaces in $\mathbb{H}^{n}(b)$ (Theorem 4.1).

\section{PRELiminAires}

2.1. The extrinsic distance. We assume throughout the paper that $P^{2}$ is a complete, noncompact, immersed, 2-dimensional submanifold in a simply connected real space form of non-positive constant sectional curvature $\mathbb{K}^{n}(b),\left(\mathbb{K}^{n}(b)=\mathbb{R}^{n}\right.$ when $b=0$ and $\mathbb{K}^{n}(b)=$ $\mathbb{H}^{n}(b)$ when $\left.b<0\right)$. All the points in these manifolds are poles. Recall that a pole is a point $o$ such that the exponential map

$$
\exp _{o}: T_{o} N^{n} \rightarrow N^{n}
$$

is a diffeomorphism. For every $x \in N^{n} \backslash\{o\}$ we define $r(x)=\operatorname{dist}_{N}(o, x)$, and this distance is realized by the length of a unique geodesic from $o$ to $x$, which is the radial geodesic from $o$. We also denote by $r$ the restriction $\left.r\right|_{P}: P \rightarrow \mathbb{R}_{+} \cup\{0\}$. This restriction is called the extrinsic distance function from $o$ in $P^{m}$. The gradients of $r$ in $N$ and $P$ are denoted by $\nabla^{N} r$ and $\nabla^{P} r$, respectively. Let us remark that $\nabla^{P} r(x)$ is just the tangential component in $P$ of $\nabla^{N} r(x)$, for all $x \in S$. Then we have the following basic relation:

$$
\nabla^{N} r=\nabla^{P} r+\left(\nabla^{N} r\right)^{\perp}
$$

where $\left(\nabla^{N} r\right)^{\perp}(x)=\nabla^{\perp} r(x)$ is perpendicular to $T_{x} P$ for all $x \in P$.

On the other hand, we should recall that all immersed surfaces $P$ in the real space forms of non-positive constant sectional curvature $N^{n}=\mathbb{K}^{n}(b)$ which satisfies $\int_{P}\left\|B^{P}\right\|^{2} d \sigma<$ 
$\infty$ are properly immersed (see [1], [20] and [21]). Therefore, we can omit the hypothesis about the properness of the immersion when we assume that $\int_{P}\left\|B^{P}\right\|^{2} d \sigma<\infty$.

Definition 2.1. Given a connected and complete surface $P^{2}$ properly immersed in a manifold $N^{n}$ with a pole $o \in N$, we denote the extrinsic metric balls of radius $t>0$ and center $o \in N$ by $D_{t}(o)$. They are defined as any connected component of the intersection

$$
B_{t}(o) \cap P=\{x \in S: r(x)<t\},
$$

where $B_{t}(o)$ denotes the open geodesic ball of radius $R$ centered at the pole $o$ in $N^{n}$.

Remark a. We want to point out that the extrinsic domains $D_{t}(o)$ are precompact sets, (because we assume in the definition above that the submanifold $P$ is properly immersed), with smooth boundary $\partial D_{t}(o)$ being a closed immersed curve in $P$. The assumption on the smoothness of $\partial D_{t}(o)$ makes no restriction. Indeed, the distance function $r$ is smooth in $\mathbb{K}^{n}(b) \backslash\{o\}$ since $\mathbb{K}^{n}(b)$ is assumed to possess a pole $o \in \mathbb{K}^{n}(b)$. Hence the restriction $\left.r\right|_{P}$ is smooth in $P$ and consequently the radii $t$ that produce smooth boundaries $\partial D_{t}(o)$ are dense in $\mathbb{R}$ by Sard's theorem and the Regular Level Set Theorem.

Remark b. When the submanifold considered is totally geodesic, namely, when $P$ is a Hyperbolic or an Euclidean subespace of the ambient real space form, the extrinsic balls become geodesic balls, and its boundary is the distance sphere. We recall here that the mean curvature of the geodesic sphere in the real space form $\mathbb{K}^{n}(b)$, 'pointed inward' is (see [22]):

$$
h_{b}(t)=\left\{\begin{array}{l}
\sqrt{b} \cot \sqrt{b} t \text { if } b>0 \\
1 / t \text { if } b=0 \\
\sqrt{-b} \operatorname{coth} \sqrt{-b} t \text { if } b<0
\end{array}\right.
$$

2.2. Hessian comparison analysis of the extrinsic distance. The 2.nd order analysis of the restricted distance function $r_{\mid P}$ defined on manifolds with a pole is firstly and foremost governed by the Hessian comparison Theorem A in [10]. We are going to give here an statement of this theorem.

Theorem 2.2 (See [10], Theorem A). Let $P^{m}$ be a complete, non-compact, properly immersed, m-dimensional submanifold in a real space form of non-positive constant sectional curvature $\mathbb{K}^{n}(b)$. Then

(i) Given $X \in T_{q} P$ unitary:

$$
\left.\operatorname{Hess}^{P}(r)(X, X)=h_{b}(r)\left(1-<X, \nabla^{N} r\right\rangle^{2}\right)+\left\langle\nabla^{N} r, B^{P}(X, X)\right\rangle
$$

where $B^{P}$ is the second fundamental form of $P$ in $N$.

(ii) Tracing equality (2.2) we obtain

$$
\Delta^{P}(r)=\left(m-\left\|\nabla^{P} r\right\|^{2}\right) h_{b}(r)+m\left\langle\nabla^{N} r, H_{P}\right\rangle,
$$

where $H_{P}$ denotes the mean curvature vector of $P$ in $N$ and $h_{b}(r)$ is the mean curvature of the geodesic $r$-spheres in $\mathbb{K}^{n}(b)$.

Let us consider now $D_{t}$ an extrinsic ball in a complete and properly immersed minimal surface $P$ in the real space form $\mathbb{K}^{n}(b)$ with $b \leq 0$. We are going to apply Gauss-Bonnet formula to the curves $\partial D_{t}$. To do that, we need to compute its geodesic curvature in the following

Proposition 2.3. Given $\partial D_{t}$ the smooth closed curves in $P$,

$$
k_{g}^{\partial D_{t}}=\frac{h_{b}(t)}{\left\|\nabla^{P} r\right\|}+<B^{P}(e, e), \frac{\nabla^{\perp} r}{\left\|\nabla^{P} r\right\|}>
$$


Proof. Let $\{e, \nu\} \subset T P$ be an orthonormal frame along the curve $\partial D_{t}$, where $e$ is the unit tangent vector to $\partial D_{t}$ and $\nu=\frac{\nabla^{P} r}{\left\|\nabla^{P}\right\|}$ is the unit normal to $\partial D_{t}$ in $P$, pointed outward.

From the definition of geodesic curvature of the extrinsic boundaries $\partial D_{t}$, we have

$$
k_{g}^{t}=-<\nabla_{e}^{P} e, \frac{\nabla^{P} r}{\left\|\nabla^{P} r\right\|}>
$$

Then, having on account the definition of Hessian

$$
\text { Hess }^{P} r(e, e)=<\nabla^{P} \nabla^{P} r, e>
$$

and the fact that $\nabla^{P} r$ and $e$ are orthogonal,

$$
k_{g}^{t}=\frac{1}{\left\|\nabla^{P} r\right\|} \text { Hess }^{P} r(e, e)
$$

Applying at this point equation (2.2) in Theorem 2.2

$$
k_{g}^{t}=\frac{1}{\left\|\nabla^{P} r\right\|}\left\{h_{b}(r)+<\nabla^{\perp} r, B^{P}(e, e)>\right\}
$$

Now, we consider $\left\{D_{t}\right\}_{t>0}$ an exhaustion of $P$ by extrinsic balls. Recall than an exhaustion of the submanifold $P$ is a sequence of subsets $\left\{D_{t} \subseteq P\right\}_{t>0}$ such that:

- $D_{t} \subseteq D_{s}$ when $s \geq t$

- $\cup_{t>0} D_{t}=P$

We have the following Corollary

Corollary 2.4. Let $P^{2}$ be an complete minimal surface immersed in a simply connected real space form with constant sectional curvature $b \leq 0, \mathbb{K}^{n}(b)$. Let us suppose that $\int_{P}\left\|B^{P}\right\|^{2} d \sigma<\infty$. Then

(i) $P$ is diffeomorphic to a compact surface $P^{*}$ punctured at a finite number of points.

(ii) For all sufficiently large $t>R_{0}>0, \chi(P)=\chi\left(D_{t}\right)$ and hence, given $\left\{D_{t}\right\}_{t>0}$ an exhaustion of $P$ by extrinsic balls,

$$
\chi(P)=\lim _{t \rightarrow \infty} \chi\left(D_{t}\right)
$$

Proof. Let us consider $\left\{D_{t}\right\}_{t>0}$ an exhaustion of $P$ by extrinsic balls, centered at the pole $p \in P$. We apply Lemma 2.4 to the smooth curves $\partial D_{t}$ : As

$$
-\left\|B^{P}\right\| \leq<B^{P}(e, e), \nabla^{\perp} r>\leq\left\|B^{P}\right\|
$$

we have, on the points of the curve $q \in \partial D_{t}$,

$$
\begin{aligned}
\left\|\nabla^{P} r\right\|(q) \cdot k_{g}^{\partial D_{t}}(q) & =h_{b}\left(r_{p}(q)\right)+<B^{P}(e, e), \nabla^{\perp} r>(q) \\
& \geq h_{b}\left(r_{p}(q)\right)-\left\|B^{P}\right\|(q)
\end{aligned}
$$

Using now Proposition 2.2 in [1], when $P^{2}$ is a cmi in $\mathbb{R}^{n}$ or Lemma 3.1 in [21], when $P^{2}$ is a cmi in $\mathbb{H}^{n}(b)$, we know that $\left\|B^{P}\right\|(q)$ goes uniformly to 0 as $t=r_{p}(q) \rightarrow \infty$. Hence, for all the points $q \in \partial D_{t}$ and for sufficiently large $t$,

$$
\left\|\nabla^{P} r\right\|(q) \cdot k_{g}^{\partial D_{t}}(q)>0
$$

Hence, $\left\|\nabla^{P} r\right\|>0$ in $\partial D_{t}$, for all sufficiently large $t$. Fixing a sufficienty large radius $R_{0}$, we can conclude that the extrinsic distance $r_{p}$ has no critical points in $P \backslash D_{R_{0}}$.

The above inequality implies that for this sufficienty large fixed radius $R_{0}$, there is a diffeomorphism

$$
\Phi: P \backslash \mathrm{D}_{R_{0}} \rightarrow \partial D_{R_{0}} \times[0, \infty[
$$


In particular, $P$ has only finitely many ends, each of finite topological type.

To proof this we apply Theorem 3.1 in [16], concluding that, as the extrinsic annuli $A_{R_{0}, R}(p)=D_{R}(p) \backslash D_{R_{0}}(p)$ contains no critical points of the extrinsic distance function $r_{p}: P \longrightarrow \mathbb{R}$ because inequality (2.9), then $D_{R}(p)$ is diffeomorphic to $D_{R_{0}}(p)$ for all $R \geq R_{0}$

The above diffeomorfism implies that we can construct $P$ from $D_{R_{0}}\left(R_{0}\right.$ big enough) attaching annulis and that $\chi\left(P \backslash D_{t}\right)=0$ when $t \geq R_{0}$. Then, for all $t>R_{0}$,

$$
\chi(P)=\chi\left(D_{t} \cup\left(P \backslash D_{t}\right)\right)=\chi\left(D_{t}\right)
$$

Corollary 2.5. Let $P^{2} \subset \mathbb{K}^{n}(b)$ be a complete minimal surface properly immersed in a real space form with curvature $b \leq 0$, let $D_{t}$ be an extrinsic disc in $P$ of radius $t>0$ and let $\partial D_{t}$ be its boundary. Then:

$$
\begin{aligned}
& -2 \pi \chi\left(D_{t}\right)+\left(b+\frac{f_{b, \alpha}^{2}(t) h_{b}(t)}{2}\right) \operatorname{Vol}\left(D_{t}\right) \\
& \quad+\left(h_{b}(t)-\frac{f_{b, \alpha}^{2}(t)}{2}\right) \int_{\partial D_{t}} \frac{1}{\left\|\nabla^{P} r\right\|} d \sigma_{t} \leq \frac{1}{2} R(t)+\frac{1}{2 f_{b, \alpha}^{2}(t)} R^{\prime}(t)
\end{aligned}
$$

where $R(t)=\int_{D_{t}}\left\|B^{P}\right\|^{2} d \sigma,\left\|B^{P}\right\|$ is the norm of the second fundamental form of $P$ in $\mathbb{K}^{n}(b), \chi\left(D_{t}\right)$ is the Euler's characterisc of $D_{t}$ and, given $\left.\alpha \in\right] 0,2[$,

$$
f_{b, \alpha}^{2}(t)=\alpha h_{b}(t)
$$

Proof. Integrating along $\partial D_{t}$ equation (2.4) and using Gauss-Bonnet theorem and co-area formula, (see [23]), we obtain

$$
\begin{aligned}
& 2 \pi \chi\left(D_{t}\right)-\int_{D_{t}} K^{P} d \sigma= \\
& h_{b}(t) \int_{\partial D_{t}} \frac{1}{\left\|\nabla^{P} r\right\|} d \sigma_{t}+\int_{\partial D_{t}}<B^{P}(e, e), \frac{\nabla^{\perp} r}{\left\|\nabla^{P} r\right\|}>d \sigma_{t}
\end{aligned}
$$

where we denote as $K^{P}$ the Gauss curvature of $P$.

But, on $\partial D_{t}$,

$$
-\left\|B^{P}\right\| \frac{\left\|\nabla^{\perp} r\right\|}{\left\|\nabla^{P} r\right\|} \leq<B^{P}(e, e), \frac{\nabla^{\perp} r}{\left\|\nabla^{P} r\right\|}>\leq\left\|B^{P}\right\| \frac{\left\|\nabla^{\perp} r\right\|}{\left\|\nabla^{P} r\right\|}
$$

so, as $f_{b, \alpha}(t) \geq 0 \forall t>0$, having into account the inequality among the arithmetic and geometric mean and applying co-area formula: 


$$
\begin{aligned}
2 & \pi \chi\left(D_{t}\right)-\int_{D_{t}} K^{P} d \sigma=h_{b}(t) \int_{\partial D_{t}} \frac{1}{\left\|\nabla^{P} r\right\|} d \sigma_{t} \\
& +\int_{\partial D_{t}}<B^{P}(e, e), \frac{\nabla^{\perp} r}{\left\|\nabla^{P} r\right\|}>d \sigma_{t} \geq h_{b}(t) \int_{\partial D_{t}} \frac{1}{\left\|\nabla^{P} r\right\|} d \sigma_{t} \\
& -\int_{\partial D_{t}}\left\|B^{P}\right\| \frac{\left\|\nabla^{\perp} r\right\|}{\left\|\nabla^{P} r\right\|} d \sigma_{t}=h_{b}(t) \int_{\partial D_{t}} \frac{1}{\left\|\nabla^{P} r\right\|} d \sigma_{t} \\
& -\int_{\partial D_{t}} \frac{\left\|B^{P}\right\|}{f_{b, \alpha}(t) \sqrt{\left\|\nabla^{P} r\right\|}} \frac{f_{b, \alpha}(t)\left\|\nabla^{\perp} r\right\|}{\sqrt{\left\|\nabla^{P} r\right\|}} d \sigma_{t} \geq h_{b}(t) \int_{\partial D_{t}} \frac{1}{\left\|\nabla^{P} r\right\|} d \sigma_{t} \\
& -\frac{1}{2} \int_{\partial D_{t}} \frac{\left\|B^{P}\right\|^{2}}{f_{b, \alpha}^{2}(r)\left\|\nabla^{P} r\right\|} d \sigma_{t}-\frac{1}{2} \int_{\partial D_{t}} \frac{f_{b, \alpha}^{2}(r)\left\|\nabla^{\perp} r\right\|^{2}}{\left\|\nabla^{P} r\right\|} d \sigma_{t} \\
\geq & h_{b}(t) \int_{\partial D_{t}} \frac{1}{\left\|\nabla^{P} r\right\|} d \sigma_{t}-\frac{1}{2 f_{b, \alpha}^{2}(t)} R^{\prime}(t)-\frac{f_{b, \alpha}^{2}(t)}{2} \int_{\partial D_{t}} \frac{\left\|\nabla^{\perp} r\right\|^{2}}{\left\|\nabla^{P} r\right\|} d \sigma_{t}
\end{aligned}
$$

Now, we apply the following lemma:

Lemma 2.6. Let $P^{2} \subset \mathbb{K}^{n}(b)$ be a surface properly immersed in a real space form with curvature $b \leq 0$, let $D_{t}$ be an extrinsic disc in $P$ of radius $t>0$ and let $\partial D_{t}$ the extrinsic circle. Then:

$$
\int_{\partial D_{t}} \frac{\left\|\nabla^{\perp} r\right\|^{2}}{\left\|\nabla^{P} r\right\|} d \sigma_{t} \leq \int_{\partial D_{t}} \frac{1}{\left\|\nabla^{P} r\right\|}-h_{b}(t) \operatorname{Vol}\left(D_{t}\right) d \sigma_{t}
$$

Proof. Applying divergence theorem and computing the Laplacian of the extrinsic distance in a minimal submanifold of a real space form (see equation (2.3) in Theorem 2.2) we have

$$
\begin{aligned}
& \int_{\partial D_{t}} \frac{\left\|\nabla^{\perp} r\right\|^{2}}{\left\|\nabla^{P} r\right\|} d \sigma_{t}=\int_{\partial D_{t}} \frac{1}{\left\|\nabla^{P} r\right\|} d \sigma_{t}-\int_{\partial D_{t}}\left\|\nabla^{P} r\right\| d \sigma_{t}=\int_{\partial D_{t}} \frac{1}{\left\|\nabla^{P} r\right\|} d \sigma_{t} \\
& -\int_{D_{t}} \Delta^{P} r d \sigma=\int_{\partial D_{t}} \frac{1}{\left\|\nabla^{P} r\right\|} d \sigma_{t}-\int_{D_{t}}\left(2-\left\|\nabla^{P} r\right\|^{2}\right) h_{b}(r) d \sigma \\
& \leq \int_{\partial D_{t}} \frac{1}{\left\|\nabla^{P} r\right\|} d \sigma_{t}-\int_{D_{t}} h_{b}(r) d \sigma \leq \int_{\partial D_{t}} \frac{1}{\left\|\nabla^{P} r\right\|} d \sigma_{t}-h_{b}(t) \operatorname{Vol}\left(D_{t}\right)
\end{aligned}
$$

Then, using inequality 2.13 in the last member of the inequalities 2.12

$$
\begin{aligned}
& 2 \pi \chi\left(D_{t}\right)-\int_{D_{t}} K^{P} d \sigma \geq\left(h_{b}(t)-\frac{f_{b, \alpha}^{2}(t)}{2}\right) \int_{\partial D_{t}} \frac{1}{\left\|\nabla^{P} r\right\|} d \sigma_{t} \\
& \quad-\frac{1}{2 f_{b, \alpha}^{2}(t)} R^{\prime}(t)+\frac{f_{b, \alpha}^{2}(t) h_{b}(t)}{2} \operatorname{Vol}\left(D_{t}\right)
\end{aligned}
$$

Now, applying Gauss equation for minimal surfaces in the real space forms $\mathbb{K}^{n}(b)$, we have

$$
\begin{aligned}
& 2 \pi \chi\left(D_{t}\right)-b \operatorname{Vol}\left(D_{t}\right)+\frac{1}{2} R(t) \geq\left(h_{b}(t)-\frac{f_{b, \alpha}^{2}(t)}{2}\right) \int_{\partial D_{t}} \frac{1}{\left\|\nabla^{P} r\right\|} d \sigma_{t} \\
& -\frac{1}{2 f_{b, \alpha}^{2}(t)} R^{\prime}(t)+\frac{f_{b, \alpha}^{2}(t) h_{b}(t)}{2} \operatorname{Vol}\left(D_{t}\right)
\end{aligned}
$$

and hence 


$$
\begin{aligned}
& -2 \pi \chi\left(D_{t}\right)+\left(b+\frac{f_{b, \alpha}^{2}(t) h_{b}(t)}{2}\right) \operatorname{Vol}\left(D_{t}\right) \\
& +\left(h_{b}(t)-\frac{f_{b, \alpha}^{2}(t)}{2}\right) \int_{\partial D_{t}} \frac{1}{\left\|\nabla^{P} r\right\|} \leq \frac{1}{2} R(t)+\frac{1}{2 f_{b, \alpha}^{2}(t)} R^{\prime}(t)
\end{aligned}
$$

\section{A UNIFIED PROOF OF CHERN-OSSERMAN INEQUALITY IN HYPERBOLIC AND EUCLIDEAN SPACES}

We are going to give a unified proof of the classical Chern-Osserman inequality for minimal surfaces of Euclidean and Hyperbolic spaces. A key outcome for this unified approach is the isoperimetric inequality stablished in [22] for the extrinsic balls of minimal submanifolds in Cartan-Hadamard manifolds, as well as a monotonicity result which is derived from it and from co-area formula, (see [18] and [2]).

Theorem C. ([22], [18], [2]) Let $P^{m}$ a complete minimal submanifold properly immersed in a Cartan-Hadamard manifold $N^{n}$ with sectional curvature $K_{N} \leq b \leq 0$. Let $D_{t}$ be an extrinsic t-ball in $P^{m}$, with center at a point $p$ which is also a pole in the ambient space $N$. Then

$$
\frac{\operatorname{Vol}\left(\partial D_{t}\right)}{\operatorname{Vol}\left(D_{t}\right)} \geq \frac{\operatorname{Vol}\left(S_{t}^{b, m-1}\right)}{\operatorname{Vol}\left(B_{t}^{b, m}\right)} \text { for all } t>0 \text {. }
$$

Furthermore, the function $f(t)=\frac{\operatorname{Vol}\left(D_{t}\right)}{\operatorname{Vol}\left(B_{t}^{b, m}\right)}$ is monotone non-decreasing in $t$.

Moreover, if equality in inequality (3.1) holds for some fixed radius $t_{0}$ then $D_{t_{0}}$ is a minimal cone in the ambient space $N^{n}$, so if $N^{n}$ is the hyperbolic space $\mathbb{K}^{n}(b), b<0$, then $P^{m}$ is totally geodesic in $\mathbb{K}^{n}(b)$.

As a consequence of Corollary 2.4 Corollary 2.5 and Theorem C, we obtain the ChernOsserman inequality for minimal submanifolds in Euclidean and Hyperbolic spaces:

Theorem 3.1. Let $P^{2}$ be an complete minimal surface immersed in a simply connected real space form with constant sectional curvature $b \leq 0, \mathbb{K}^{n}(b)$. Let us suppose that $\int_{P}\left\|B^{P}\right\|^{2} d \sigma<\infty$. Then

(1) P has finite topological type.

(2) $\operatorname{Sup}_{t>0}\left(\frac{\operatorname{Vol}\left(D_{t}\right)}{\operatorname{Vol}\left(B_{t}^{b, 2}\right)}\right)<\infty$

$$
\text { (3) }-\chi(P) \leq \frac{\int_{P}\left\|B^{P}\right\|^{2}}{4 \pi}-\operatorname{Sup}_{t>0} \frac{\operatorname{Vol}\left(D_{t}\right)}{\operatorname{Vol}\left(B_{t}^{b, 2}\right)}
$$

where $\chi(P)$ is the Euler characteristic of $P$.

Remark c. We should note the following technical observation: to prove the result when the ambient space is the Hyperbolic space $\mathbb{H}^{n}(b)$ it is necessary to consider a suitable exhaustion of $P^{2} \subseteq \mathbb{H}^{n}(b)$ by extrinsic balls $\left\{D_{t_{i}}\right\}_{i=1}^{\infty}$ such that their radius $\left\{t_{i}\right\}_{i=1}^{\infty}$ determines a subsequence $\left\{\frac{R^{\prime}\left(t_{i}\right)}{h_{b}\left(t_{i}\right)}\right\}_{i=1}^{\infty}$ which converges to zero by virtue of the fact that, in this case, $\int_{P} \frac{\left\|B^{P}\right\|^{2}}{h_{b}(t)} d \sigma<\infty$.

However, when we consider the surface $P$ immersed in the Euclidean space, we shall use the estimate of the curvature decay obtained by Anderson in [1], which holds for any monotone increasing sequence of radius $\left\{t_{i}\right\}_{i=1}^{\infty}$.

Proof. We are going to divide the proof in two cases: the Case I, where the ambient space is the Hyperbolic space $\mathbb{H}^{n}(b)$, and the Case II where the ambient space is the Euclidean space $\mathbb{R}^{n}$. 
Case I. Let us consider $P$ (properly) immersed in $\mathbb{H}^{n}(b)$. Let $\left\{D_{t}\right\}_{t>0}$ be an exhaustion of $P$ by extrinsic balls. Using co-area formula, we know that

$$
\frac{d}{d t} \operatorname{Vol}\left(D_{t}\right)=\int_{\partial D_{t}} \frac{1}{\left\|\nabla^{P} r\right\|} d \sigma_{t}
$$

Hence, applying Corollary 2.5 we have

$$
\begin{aligned}
& -2 \pi \chi\left(D_{t}\right)+\left(b+\frac{f_{b, \alpha}^{2}(t) h_{b}(t)}{2}\right) \operatorname{Vol}\left(D_{t}\right) \\
& +\left(h_{b}(t)-\frac{f_{b, \alpha}^{2}(t)}{2}\right) \frac{d}{d t} \operatorname{Vol}\left(D_{t}\right) \leq \frac{1}{2} R(t)+\frac{1}{2 f_{b, \alpha}^{2}(t)} R^{\prime}(t)
\end{aligned}
$$

On the other hand, from 3.2, $\frac{d}{d t} \operatorname{Vol}\left(D_{t}\right) \geq \operatorname{Vol}\left(\partial D_{t}\right)$. Therefore, using inequality (3.3) we obtain

$$
\begin{aligned}
& -2 \pi \chi\left(D_{t}\right) \\
& +\operatorname{Vol}\left(D_{t}\right)\left[\left(b+\frac{f_{b, \alpha}^{2}(t) h_{b}(t)}{2}\right)+\left(h_{b}(t)-\frac{f_{b, \alpha}^{2}(t)}{2}\right) \frac{\operatorname{Vol}\left(\partial D_{t}\right)}{\operatorname{Vol}\left(D_{t}\right)}\right] \\
& \leq \frac{1}{2} R(t)+\frac{1}{2 f_{b, \alpha}^{2}(t)} R^{\prime}(t)
\end{aligned}
$$

Applying isoperimetric inequality (3.1) in Theorem $\mathrm{C}$ we have

$$
\begin{aligned}
& -2 \pi \chi\left(D_{t}\right) \\
& +\operatorname{Vol}\left(D_{t}\right)\left[\left(b+\frac{f_{b, \alpha}^{2}(t) h_{b}(t)}{2}\right)+\left(h_{b}(t)-\frac{f_{b, \alpha}^{2}(t)}{2}\right) \frac{\operatorname{Vol}\left(S_{t}^{b, 1}\right)}{\operatorname{Vol}\left(B_{t}^{b, 2}\right)}\right] \\
& \leq \frac{1}{2} R(t)+\frac{1}{2 f_{b, \alpha}^{2}(t)} R^{\prime}(t)
\end{aligned}
$$

Hence, using the fact that

$$
b \operatorname{Vol}\left(B_{t}^{b, 2}\right)+h_{b}(t) \operatorname{Vol}\left(S_{t}^{b, 1}=2 \pi \forall t>0\right.
$$

we have

$$
\begin{aligned}
& -2 \pi \chi\left(D_{t}\right) \\
& +\frac{\operatorname{Vol}\left(D_{t}\right)}{\operatorname{Vol}\left(B_{t}^{b, 2}\right)}\left[2 \pi+\frac{f_{b, \alpha}^{2}(t) h_{b}(t)}{2} \operatorname{Vol}\left(B_{t}^{b, 2}\right)-\frac{f_{b, \alpha}^{2}(t)}{2} \operatorname{Vol}\left(S_{t}^{b, 1}\right)\right] \\
& \leq \frac{1}{2} R(t)+\frac{1}{2 f_{b, \alpha}^{2}(t)} R^{\prime}(t)
\end{aligned}
$$

and therefore

$$
\begin{aligned}
& -2 \pi \chi\left(D_{t}\right)+\frac{\operatorname{Vol}\left(D_{t}\right)}{\operatorname{Vol}\left(B_{t}^{b, 2}\right)}\left[2 \pi-2 \pi \frac{f_{b, \alpha}^{2}(t)}{2} \frac{\operatorname{Vol}\left(B_{t}^{b, 2}\right)}{\operatorname{Vol}\left(S_{t}^{b, 1}\right)}\right] \\
\leq & \frac{1}{2} R(t)+\frac{1}{2 f_{b, \alpha}^{2}(t)} R^{\prime}(t)
\end{aligned}
$$

Therefore, for all $t>0$,

$$
\begin{gathered}
\frac{\operatorname{Vol}\left(D_{t}\right)}{\operatorname{Vol}\left(B_{t}^{b, 2}\right)}\left(1-\frac{\alpha h_{b}(t)}{2} \frac{\operatorname{Vol}\left(B_{t}^{b, 2}\right)}{\operatorname{Vol}\left(S_{t}^{b, 1}\right)}\right)-\chi\left(D_{t}\right) \\
\leq \frac{R(t)}{4 \pi}+\frac{R^{\prime}(t)}{4 \pi \alpha h_{b}(t)}
\end{gathered}
$$


As $\frac{\left\|B^{P}\right\|^{2}}{h_{b}(t)} \leq \frac{1}{\sqrt{-b}}\left\|B^{P}\right\|^{2}$, then $\int_{P}\left\|B^{P}\right\|^{2} d \sigma<\infty$ implies $\int_{P} \frac{\left\|B^{P}\right\|^{2}}{h_{b}(t)} d \sigma<\infty$. Hence, by co-area formula:

$$
\int_{0}^{\infty}\left(\int_{D_{t}} \frac{\left\|B^{P}\right\|^{2}}{\left\|\nabla^{P} r\right\| h_{b}(r)}\right) d t=\int_{0}^{\infty}\left(\frac{R^{\prime}(t)}{h_{b}(t)}\right) d t<\infty
$$

Therefore, there is a monotone increasing (sub)sequence $\left\{t_{i}\right\}_{i=1}^{\infty}$ tending to infinity, (namely, $t_{i} \rightarrow \infty$ when $i \rightarrow \infty$ ), such that $\frac{R^{\prime}\left(t_{i}\right)}{h_{b}\left(t_{i}\right)} \rightarrow 0$ when $i \rightarrow \infty$.

Let us consider the exhaustion of $P$ by these extrinsic balls, namely, $\left\{D_{t_{i}}\right\}_{i=1}^{\infty}$. Then we have, replacing $t$ for $t_{i}$ and taking limits when $i \rightarrow \infty$ in inequality (3.8) and applying Corollary 2.4(ii),

$$
\begin{aligned}
\operatorname{Sup}_{i} & \frac{\operatorname{Vol}\left(D_{t_{i}}\right)}{\operatorname{Vol}\left(B_{t_{i}}^{b, 2}\right)}\left(1-\frac{\alpha}{2}\right)-\chi(P) \\
\leq & \lim _{i \rightarrow \infty} \frac{R\left(t_{i}\right)}{4 \pi}=\frac{1}{4 \pi} \int_{P}\left\|B^{P}\right\|^{2} d \sigma<\infty
\end{aligned}
$$

for all $\alpha$ such that $0<\alpha<2$.

Hence, as $\frac{\operatorname{Vol}\left(D_{t}\right)}{\operatorname{Vol}\left(B_{t}^{b, 2}\right)}$ is a continuous non decreasing function of $t$, we can conclude that $\operatorname{Sup}_{t>0} \frac{\operatorname{Vol}\left(D_{t}\right)}{\operatorname{Vol}\left(B_{t}^{b, 2}\right)}<\infty$ and $-\chi(P)<\infty$.

Then, letting $\alpha$ tend to 0 in (3.10), we get, for all $t>0$ :

$$
\operatorname{Sup}_{t>0} \frac{\operatorname{Vol}\left(D_{t}\right)}{\operatorname{Vol}\left(B_{t}^{b, 2}\right)}-\chi(P) \leq \frac{\int_{P}\left\|B^{P}\right\|^{2}}{4 \pi}
$$

Case II. Let us consider $P$ immersed in $\mathbb{R}^{n}$. We consider, as in the proof above, an exhaustion of $P$ by extrinsic balls, $\left\{D_{t}\right\}_{t>0}$, but now, and following [1], these extrinsic balls will be centered at the origin $0 \in \mathbb{R}^{n}$, which we assume, without loss of generality, that belongs to the surface $P$. Applying Corollary 2.5 we have

$$
\begin{aligned}
-2 \pi \chi & \left(D_{t}\right)+\left(\frac{\alpha}{2 t^{2}}\right) \operatorname{Vol}\left(D_{t}\right) \\
& +\left(\frac{1}{t}-\frac{\alpha}{2 t}\right) \int_{\partial D_{t}} \frac{1}{\left\|\nabla^{P} r\right\|} \leq \frac{1}{2} R(t)+\frac{t}{2 \alpha} R^{\prime}(t)
\end{aligned}
$$

Now, as $\int_{P}\left\|B^{P}\right\|^{2} d \sigma<\infty$, we can apply Proposition 2.2 in [1], so we have, for $\alpha \in] 0,2[$,

$$
\frac{t}{2 \alpha} R^{\prime}(t)=\frac{t}{2 \alpha} \int_{\partial D_{t}} \frac{\left\|B^{P}\right\|^{2}}{\left\|\nabla^{P} r\right\|} d \sigma \leq \frac{\mu(t)}{2 \alpha t} \int_{\partial D_{t}} \frac{1}{\left\|\nabla^{P} r\right\|} d \sigma
$$

being $\mu(t)$ such that $\lim _{t \rightarrow \infty} \mu(t)=0$ and therefore, from (3.12),

$$
\begin{aligned}
& -2 \pi \chi\left(D_{t}\right)+\operatorname{Vol}\left(D_{t}\right)\left(\frac{\alpha}{2 t^{2}}\right) \\
& +\left(\frac{1}{t}-\frac{\alpha}{2 t}-\frac{\mu(t)}{2 \alpha t}\right) \int_{\partial D_{t}} \frac{1}{\left\|\nabla^{P} r\right\|} d \sigma_{t} \leq \frac{1}{2} R(t)
\end{aligned}
$$

On the other hand, $\frac{1}{t}-\frac{\alpha}{2 t}-\frac{\mu(t)}{2 \alpha t} \geq 0$ if and only if $\mu(t) \leq \alpha(2-\alpha)$, which it is true for $t$ big enough, namely, for $t>t_{\alpha}$ because $\lim _{t \rightarrow \infty} \mu(t)=0$. Hence, as $\operatorname{Vol}\left(\partial D_{t}\right) \leq$ $\int_{\partial D_{t}} \frac{1}{\left\|\nabla^{P} r\right\|} d \sigma_{t}$, inequality (3.14) becomes, for all $t>t_{\alpha}$ 


$$
\begin{aligned}
& -2 \pi \chi\left(D_{t}\right) \\
& +\operatorname{Vol}\left(D_{t}\right)\left[\frac{1}{t}\left(1-\frac{\alpha}{2}-\frac{\mu(t)}{2 \alpha}\right) \frac{\operatorname{Vol}\left(\partial D_{t}\right)}{\operatorname{Vol}\left(D_{t}\right)}+\frac{\alpha}{2 t^{2}}\right] \leq \frac{1}{2} R(t)
\end{aligned}
$$

and, applying inequality (3.1) in Theorem $\mathrm{C}$, we have, for all $t>t_{\alpha}$

$$
\begin{aligned}
& -2 \pi \chi\left(D_{t}\right) \\
& +\frac{\operatorname{Vol}\left(D_{t}\right)}{\operatorname{Vol}\left(B_{t}^{0,2}\right)}\left[2 \pi\left(1-\frac{\alpha}{2}-\frac{\mu(t)}{2 \alpha}\right)+\frac{\pi \alpha}{2}\right] \leq \frac{1}{2} R(t)
\end{aligned}
$$

Then, taking limits when $t \rightarrow \infty$ in inequality (3.16) and applying Corollary 2.4, we have that $\lim _{t \rightarrow \infty} \mu(t)=0$ and $\chi(P)=\lim _{t \rightarrow \infty} \chi\left(D_{t}\right)$, so we obtain, for all $\alpha$ such that $0<\alpha<2$ :

$$
\begin{aligned}
2 \pi \operatorname{Sup}_{t} \frac{\operatorname{Vol}\left(D_{t}\right)}{\operatorname{Vol}\left(B_{t}^{0,2}\right)} & \left(1-\frac{\alpha}{2}+\frac{\pi \alpha}{2}\right) \\
& -2 \pi \chi(P) \leq \frac{\int_{P}\left\|B^{P}\right\|^{2}}{2}<\infty
\end{aligned}
$$

Therefore we obtain $\operatorname{Sup}_{t>0} \frac{\operatorname{Vol}\left(D_{t}\right)}{\operatorname{Vol}\left(B_{t}^{0,2}\right)}<\infty$ and $-\chi(P)<\infty$.

Then, letting $\alpha$ tend to 0 we obtain, for all $t>0$ :

$$
\operatorname{Sup}_{t>0} \frac{\operatorname{Vol}\left(D_{t}\right)}{\operatorname{Vol}\left(B_{t}^{0,2}\right)}-\chi(P) \leq \frac{\int_{P}\left\|B^{P}\right\|^{2}}{4 \pi}
$$

As a corollary of the Chern-Osserman inequality in $\mathbb{R}^{n}$, we have the following results about the asymptotic behavior of the $\infty$-isoperimetric quotient of extrinsic balls. We note that in the paper [25] it was proved the following asymptotic behavior (described in equation (3.19) of Corollary 3.2 and in equation (1) of Corollary 3.3) for a family of domains in $\mathbb{H}^{2}(b),\left\{C_{t}\right\}_{t>0}$, depending upon a parameter $t \in \mathbb{R}^{+}$, which are convex with respect to horocycles and expands over the whole Hyperbolic plane. We have, in this context,

$$
\lim _{t \rightarrow \infty} \frac{\operatorname{Perimeter}\left(C_{t}\right)}{\operatorname{Area}\left(C_{t}\right)}=\sqrt{-b}
$$

Our first result, in the Euclidean setting is

Corollary 3.2. Let $P^{2}$ be an complete minimal surface immersed in $\mathbb{R}^{n}$. Let us suppose that $\int_{P}\left\|B^{P}\right\|^{2} d \sigma<\infty$. Then for every exhaustion of $P$ with extrinsic balls:

$$
\lim _{t \rightarrow \infty} \frac{\operatorname{Vol}\left(\partial D_{t}\right)}{\operatorname{Vol}\left(D_{t}\right)}=0
$$

Proof. Now, we consider a minimal surface $P$ properly immersed in the Euclidean space $\mathbb{R}^{n}$. By inequality (3.14) we can write, as $\operatorname{Vol}\left(S_{t}^{0,1}\right)=2 \pi t$ :

$$
\frac{\left(\operatorname{Vol}\left(D_{t}\right)\right)^{\prime}}{\operatorname{Vol}\left(S_{t}^{0,1}\right)} \leq \frac{\frac{R(t)}{4 \pi}+\chi\left(D_{t}\right)-\frac{\alpha}{4} \frac{\operatorname{Vol}\left(D_{t}\right)}{\operatorname{Vol}\left(B_{t}^{0,2}\right)}}{1-\frac{\alpha}{2}-\frac{\mu(t)}{2 \alpha}}
$$

Hence, as we know, on the other hand, that

$$
\left(\frac{\operatorname{Vol}\left(D_{t}\right)}{\operatorname{Vol}\left(B_{t}^{0,2}\right)}\right)^{\prime}=\frac{\operatorname{Vol}\left(S_{t}^{0,1}\right)}{\operatorname{Vol}\left(B_{t}^{0,2}\right)}\left(\frac{\left(\operatorname{Vol}\left(D_{t}\right)\right)^{\prime}}{\operatorname{Vol}\left(S_{t}^{0,1}\right)}-\frac{\operatorname{Vol}\left(D_{t}\right)}{\operatorname{Vol}\left(B_{t}^{0,2}\right)}\right)
$$

then 


$$
\left(\frac{\operatorname{Vol}\left(D_{t}\right)}{\operatorname{Vol}\left(B_{t}^{0,2}\right)}\right)^{\prime} \leq \frac{\operatorname{Vol}\left(S_{t}^{0,1}\right)}{\operatorname{Vol}\left(B_{t}^{0,2}\right)}\left(\frac{\frac{R(t)}{4 \pi}+\chi\left(D_{t}\right)-\frac{\alpha}{4} \frac{\operatorname{Vol}\left(D_{t}\right)}{\operatorname{Vol}\left(B_{t}\right)}}{1-\frac{\alpha}{2}-\frac{\mu(t)}{2 \alpha}}-\frac{\operatorname{Vol}\left(D_{t}\right)}{\operatorname{Vol}\left(B_{t}^{0,2}\right)}\right)
$$

As, on the other hand,

$$
\left(\frac{\operatorname{Vol}\left(D_{t}\right)}{\operatorname{Vol}\left(B_{t}^{0,2}\right)}\right)^{\prime} \geq \frac{\operatorname{Vol}\left(D_{t}\right)}{\operatorname{Vol}\left(B_{t}^{0,2}\right)}\left(\frac{\operatorname{Vol}\left(\partial D_{t}\right)}{\operatorname{Vol}\left(D_{t}\right)}-\frac{\operatorname{Vol}\left(S_{t}^{0,1}\right)}{\operatorname{Vol}\left(B_{t}^{0,2}\right)}\right) \geq 0
$$

putting all together:

$$
\begin{aligned}
& 0 \leq \frac{\operatorname{Vol}\left(D_{t}\right)}{\operatorname{Vol}\left(B_{t}^{0,2}\right)}\left(\frac{\operatorname{Vol}\left(\partial D_{t}\right)}{\operatorname{Vol}\left(D_{t}\right)}-\frac{\operatorname{Vol}\left(S_{t}^{0,1}\right)}{\operatorname{Vol}\left(B_{t}^{0,2}\right)}\right) \leq\left(\frac{\operatorname{Vol}\left(D_{t}\right)}{\operatorname{Vol}\left(B_{t}^{0,2}\right)}\right)^{\prime} \leq \\
& \frac{\operatorname{Vol}\left(S_{t}^{0,1}\right)}{\operatorname{Vol}\left(B_{t}^{0,2}\right)}\left(\frac{\frac{R(t)}{4 \pi}+\chi\left(D_{t}\right)-\frac{\alpha}{4} \frac{\operatorname{Vol}\left(D_{t}\right)}{\operatorname{Vol}\left(B_{t}\right)}}{1-\frac{\alpha}{2}-\frac{\mu(t)}{2 \alpha}}-\frac{\operatorname{Vol}\left(D_{t}\right)}{\operatorname{Vol}\left(B_{t}^{0,2}\right)}\right)
\end{aligned}
$$

Then taking limits $t \rightarrow \infty$, and considering that, thanks to Theorem 3.1

$$
\lim _{t \rightarrow \infty} \frac{\operatorname{Vol}\left(S_{t}^{0,1}\right)}{\operatorname{Vol}\left(B_{t}^{0,2}\right)}\left(\frac{\frac{R(t)}{4 \pi}+\chi\left(D_{t}\right)-\frac{\alpha}{4} \frac{\operatorname{Vol}\left(D_{t}\right)}{\operatorname{Vol}\left(B_{t}^{0,2}\right)}}{1-\frac{\alpha}{2}-\frac{\mu(t)}{2 \alpha}}-\frac{\operatorname{Vol}\left(D_{t}\right)}{\operatorname{Vol}\left(B_{t}^{0,2}\right)}\right)=0
$$

we conclude:

$$
\lim _{t \rightarrow \infty} \frac{\operatorname{Vol}\left(\partial D_{t}\right)}{\operatorname{Vol}\left(D_{t}\right)}=0
$$

and

$$
\lim _{t \rightarrow \infty}\left(\frac{\operatorname{Vol}\left(D_{t}\right)}{\operatorname{Vol}\left(B_{t}^{0,2}\right)}\right)^{\prime}=0
$$

As a consequence of Remark c in the Hyperbolic setting Corollary 3.2 holds only for a suitable exhaustion of $P$ by extrinsic balls:

Corollary 3.3. Let $P^{2}$ be an complete minimal surface (properly) immersed in the Hyperbolic space, $\mathbb{H}^{n}(b)$. Let us suppose that $\int_{P}\left\|B^{P}\right\|^{2} d \sigma<\infty$. Then, there exist at least one exhaustion of $P$ by extrinsic balls $\left\{D_{t_{i}}\right\}_{i=0}^{\infty}$ (where $t_{i} \rightarrow \infty$ when $i \rightarrow \infty$ ) such that:
(1) $\lim _{i \rightarrow \infty} \frac{\operatorname{Vol}\left(\partial D_{t_{i}}\right)}{\operatorname{Vol}\left(D_{t_{i}}\right)}=\sqrt{-b}$
(2) $\lim _{i \rightarrow \infty} \frac{\left(\operatorname{Vol}\left(D_{t_{i}}\right)\right)^{\prime}}{\operatorname{Vol}\left(S_{t_{i}}^{b, 1}\right)}=\lim _{i \rightarrow \infty} \frac{\operatorname{Vol}\left(D_{t_{i}}\right)}{\operatorname{Vol}\left(B_{t_{i}}^{b, 2}\right)}$
(3) $\lim _{i \rightarrow \infty} \frac{\operatorname{Vol}\left(\partial D_{t_{i}}\right)}{\operatorname{Vol}\left(S_{t_{i}}^{b, 1}\right)}=\lim _{i \rightarrow \infty} \frac{\operatorname{Vol}\left(D_{t_{i}}\right)}{\operatorname{Vol}\left(B_{t_{i}}^{b, 2}\right)}$

Proof. Let us define

$$
D(t):=\frac{\operatorname{Vol}\left(D_{t}\right)^{\prime}}{\operatorname{Vol}\left(D_{t}\right)}-\frac{\operatorname{Vol}\left(S_{t}^{b, 1}\right)}{\operatorname{Vol}\left(B_{t}^{b, 2}\right)}=\left[\ln \left(\frac{\operatorname{Vol}\left(D_{t}\right)}{\operatorname{Vol}\left(B_{t}\right)}\right)\right]^{\prime}
$$

It is easy to see by the co-area formula and Theorem $\mathrm{C}$ that $D(t)$ is a nonnegative function. Integrating between $t_{0}>0$ and $t$ :

$$
\frac{\operatorname{Vol}\left(D_{t}\right)}{\operatorname{Vol}\left(B_{t}\right)}=\frac{\operatorname{Vol}\left(D_{t_{0}}\right)}{\operatorname{Vol}\left(B_{t_{0}}\right)} e^{\int_{t_{0}}^{t} D(s) d s}
$$


But on the other hand by Theorem 3.1 we know that $\lim _{t \rightarrow \infty} \frac{\operatorname{Vol}\left(D_{t}\right)}{\operatorname{Vol}\left(B_{t}\right)}=\sup _{t} \frac{\operatorname{Vol}\left(D_{t}\right)}{\operatorname{Vol}\left(B_{t}\right)}<\infty$. Then:

$$
\int_{t_{0}}^{\infty} D(s) d s<\infty
$$

and hence there is a monotone increasing sequence $\left\{t_{i}\right\}_{i=0}^{\infty}$ tending to infinity, such that:

$$
\lim _{i \rightarrow \infty} D\left(t_{i}\right)=0
$$

Observe that the above limit implies that $\lim _{i \rightarrow \infty} \frac{\left(\operatorname{Vol}\left(D_{t_{i}}\right)\right)^{\prime}}{\operatorname{Vol}\left(D_{t_{i}}\right)}=\sqrt{-b}$.

Moreover, by Theorem $\mathrm{C}$

$$
\begin{aligned}
D(t) & =\frac{\operatorname{Vol}\left(B_{t_{i}}^{b, 2}\right)}{\operatorname{Vol}\left(D_{t_{i}}\right)} \frac{\operatorname{Vol}\left(S_{t_{i}}^{b, 1}\right)}{\operatorname{Vol}\left(B_{t_{i}}^{b, 2}\right)}\left(\frac{\left(\operatorname{Vol}\left(D_{t_{i}}\right)\right)^{\prime}}{\operatorname{Vol}\left(S_{t_{i}}^{b, 1}\right)}-\frac{\operatorname{Vol}\left(D_{t_{i}}\right)}{\operatorname{Vol}\left(B_{t_{i}}^{b, 2}\right)}\right) \\
& \geq \frac{\operatorname{Vol}\left(B_{t_{i}}^{b, 2}\right)}{\operatorname{Vol}\left(D_{t_{i}}\right)} \frac{\operatorname{Vol}\left(S_{t_{i}}^{b, 1}\right)}{\operatorname{Vol}\left(B_{t_{i}}^{b, 2}\right)}\left(\frac{\operatorname{Vol}\left(\partial D_{t_{i}}\right)}{\operatorname{Vol}\left(S_{t_{i}}^{b, 1}\right)}-\frac{\operatorname{Vol}\left(D_{t_{i}}\right)}{\operatorname{Vol}\left(B_{t_{i}}^{b, 2}\right)}\right) \geq 0
\end{aligned}
$$

then using (3.25) and that $\lim _{t \rightarrow \infty} \frac{\operatorname{Vol}\left(B_{t}^{b, 2}\right)}{\operatorname{Vol}\left(D_{t}\right)}<\infty$ and $\lim _{i \rightarrow \infty} \frac{\left(\operatorname{Vol}\left(D_{t_{i}}\right)\right)^{\prime}}{\operatorname{Vol}\left(D_{t_{i}}\right)}=\sqrt{-b}$ we have:

$$
\begin{aligned}
\lim _{i \rightarrow \infty} \frac{\left(\operatorname{Vol}\left(D_{t_{i}}\right)\right)^{\prime}}{\operatorname{Vol}\left(S_{t_{i}}^{b, 1}\right)}=\lim _{i \rightarrow \infty} \frac{\operatorname{Vol}\left(D_{t_{i}}\right)}{\operatorname{Vol}\left(B_{t_{i}}^{b, 2}\right)} \\
\lim _{i \rightarrow \infty} \frac{\operatorname{Vol}\left(\partial D_{t_{i}}\right)}{\operatorname{Vol}\left(S_{t_{i}}^{b, 1}\right)}=\lim _{i \rightarrow \infty} \frac{\operatorname{Vol}\left(D_{t_{i}}\right)}{\operatorname{Vol}\left(B_{t_{i}}^{b, 2}\right)}
\end{aligned}
$$

And finally since:

$$
D\left(t_{i}\right) \geq \frac{\operatorname{Vol}\left(\partial D_{t_{i}}\right)}{\operatorname{Vol}\left(D_{t_{i}}\right)}-\frac{\operatorname{Vol}\left(S_{t_{i}}^{b, 1}\right)}{\operatorname{Vol}\left(B_{t_{i}}^{b, 2}\right)} \geq 0
$$

we obtain, having into acount again (3.25):

$$
\lim _{i \rightarrow \infty} \frac{\operatorname{Vol}\left(\partial D_{t_{i}}\right)}{\operatorname{Vol}\left(D_{t_{i}}\right)}=\sqrt{-b}
$$

\section{A Chern-OSERman TYPe EQUALity FOR Minimal SURFACES IN HyPERBoliC} SPACES

In this section we are going to see that complete minimal surfaces properly immersed in Hyperbolic spaces of finite total extrinsic curvature satisfies a Chern-Osserman's type equality.

Theorem 4.1. Let $P^{2}$ be a complete immersed minimal surface in $\mathbb{H}^{n}(b)$. Let us suppose that $\int_{P}\left\|B^{P}\right\|^{2} d \sigma<\infty$. then

$$
-\chi(P)=\frac{1}{4 \pi} \int_{P}\left\|B^{P}\right\|^{2}-\operatorname{Sup}_{t>0} \frac{\operatorname{Vol}\left(D_{t}\right)}{\operatorname{Vol}\left(B_{t}^{b, 2}\right)}-\frac{1}{2 \pi} G_{b}(P)
$$

where $G_{b}(P)$ is a nonnegative quantity which do not depends on the exahustion $\left\{D_{t}\right\}_{t>0}$ of $P$ and is given by

$$
\begin{aligned}
G_{b}(P) & :=\lim _{t \rightarrow \infty}\left(h_{b}(t) \operatorname{Vol}\left(B_{t}^{b, 2}\right)\left(\frac{\left(\operatorname{Vol}\left(D_{t}\right)\right)}{\operatorname{Vol}\left(B_{t}^{b, 2}\right)}\right)^{\prime}\right. \\
& \left.+\int_{\partial D_{t}}<B^{P}(e, e), \frac{\nabla^{\perp} r}{\left\|\nabla^{P} r\right\|}>d \sigma_{t}\right)
\end{aligned}
$$


Proof. In Corollary 2.4, it was obtained a sufficienty large radius $R_{0}$, such that the extrinsic distance $r_{p}$ has no critical points in $P \backslash \mathrm{D}_{R_{0}}$.

Hence for this sufficienty large fixed radius $R_{0}$, there is a diffeomorphism

$$
\Phi: P \backslash \mathrm{D}_{R_{0}} \rightarrow \partial D_{R_{0}} \times[0, \infty[
$$

so, in particular, $P$ has only finitely many ends, each of finite topological type.

The above diffeomorfism implied that we could construct $P$ from $D_{R_{0}}$ ( $R_{0}$ big enough) attaching annulis and that $\chi\left(P \backslash D_{t}\right)=0$ when $t \geq R_{0}$, and hence for all $t>R_{0}$, $\chi(P)=\chi\left(D_{t}\right)$.

Let us consider now an exhaustion by extrinsic balls $\left\{D_{t}\right\}_{t>0}$ of $P$ such that the extrinsic distance $r_{p}$ has no critical points in $P \backslash \mathrm{D}_{R_{0}}$.

Applying now Gauss-Bonnet Theorem to the extrinsic balls $D_{t}$

$$
2 \pi \chi(P)=\int_{D_{t}} K_{P} d \sigma+\int_{\partial D_{t}} k_{g} d \sigma_{t}
$$

Having in to account equation (2.4) and the Gauss formula, we have, for all sufficiently large radius $t>R_{0}$

$$
\begin{aligned}
2 \pi \chi(P) & =-\frac{1}{2} \int_{D_{t}}\left\|B^{P}\right\|^{2}+b \operatorname{Vol}\left(D_{t}\right)+h_{b}(t)\left(\operatorname{Vol}\left(D_{t}\right)\right)^{\prime} \\
& +\int_{\partial D_{t}}<B^{P}(e, e), \frac{\nabla^{\perp} r}{\left\|\nabla^{P} r\right\|}>d \sigma_{t}=-\frac{1}{2} \int_{D_{t}}\left\|B^{P}\right\|^{2} d \sigma \\
& +\frac{\operatorname{Vol}\left(D_{t}\right)}{\operatorname{Vol}\left(B_{t}^{b, 2}\right)}\left(b \cdot \operatorname{Vol}\left(B_{t}^{b, 2}\right)+h_{b}(t)\left(\operatorname{Vol}\left(D_{t}\right)\right)^{\prime} \frac{\operatorname{Vol}\left(B_{t}^{b, 2}\right)}{\operatorname{Vol}\left(D_{t}\right)}\right. \\
& \left.+\frac{\operatorname{Vol}\left(B_{t}^{b, 2}\right)}{\operatorname{Vol}\left(D_{t}\right)} \int_{\partial D_{t}}<B^{P}(e, e), \frac{\nabla^{\perp} r}{\left\|\nabla^{P} r\right\|}>d \sigma_{t}\right)
\end{aligned}
$$

But $2 \pi=b \cdot \operatorname{Vol}\left(B_{t}^{b, 2}\right)+h_{b}(t) \operatorname{Vol}\left(S_{t}^{b, 1}\right) \forall t>0$, so, for all sufficiently large radius $t>R_{0}$ :

$$
\begin{aligned}
2 \pi \chi(P)=-\frac{1}{2} & \int_{D_{t}}\left\|B^{P}\right\|^{2} d \sigma+\frac{\operatorname{Vol}\left(D_{t}\right)}{\operatorname{Vol}\left(B_{t}^{b, 2}\right)}\left(2 \pi-h_{b}(t) \operatorname{Vol}\left(S_{t}^{b, 1}\right)+h_{b}(t)\left(\operatorname{Vol}\left(D_{t}\right)\right)^{\prime} \frac{\operatorname{Vol}\left(B_{t}^{b, 2}\right)}{\operatorname{Vol}\left(D_{t}\right)}\right. \\
& \left.+\frac{\operatorname{Vol}\left(B_{t}^{b, 2}\right)}{\operatorname{Vol}\left(D_{t}\right)} \int_{\partial D_{t}}<B^{P}(e, e), \frac{\nabla^{\perp} r}{\left\|\nabla^{P} r\right\|}>d \sigma_{t}\right)=-\frac{1}{2} \int_{D_{t}}\left\|B^{P}\right\|^{2} d \sigma \\
& +\frac{\operatorname{Vol}\left(D_{t}\right)}{\operatorname{Vol}\left(B_{t}^{b, 2}\right)}\left(2 \pi+h_{b}(t) \operatorname{Vol}\left(S_{t}^{b, 1}\right)\left\{\frac{\operatorname{Vol}\left(B_{t}^{b, 2}\right)}{\operatorname{Vol}\left(D_{t}\right)} \frac{\left(\operatorname{Vol}\left(D_{t}\right)\right)^{\prime}}{\operatorname{Vol}\left(S_{t}^{b, 1}\right)}-1\right\}\right. \\
& \left.+\frac{\operatorname{Vol}\left(B_{t}^{b, 2}\right)}{\operatorname{Vol}\left(D_{t}\right)} \int_{\partial D_{t}}<B^{P}(e, e), \frac{\nabla^{\perp} r}{\left\|\nabla^{P} r\right\|}>d \sigma_{t}\right)=-\frac{1}{2} \int_{D_{t}}\left\|B^{P}\right\|^{2} d \sigma \\
& +2 \pi \frac{\operatorname{Vol}\left(D_{t}\right)}{\operatorname{Vol}\left(B_{t}^{b, 2}\right)}+h_{b}(t) \operatorname{Vol}\left(S_{t}^{b, 1}\right)\left\{\frac{\left(\operatorname{Vol}\left(D_{t}\right)\right)^{\prime}}{\operatorname{Vol}\left(S_{t}^{b, 1}\right)}-\frac{\operatorname{Vol}\left(D_{t}\right)}{\operatorname{Vol}\left(B_{t}^{b, 2}\right)}\right\} \int_{\partial D_{t}}<B^{P}(e, e), \frac{\nabla^{\perp} r}{\left\|\nabla^{P} r\right\|}>d \sigma_{t} \\
& =-\frac{1}{2} \int_{D_{t}}\left\|B^{P}\right\|^{2} d \sigma+2 \pi \frac{\operatorname{Vol}\left(D_{t}\right)}{\operatorname{Vol}\left(B_{t}^{b, 2}\right)}+h_{b}(t) \operatorname{Vol}\left(B_{t}^{b, 2}\right)\left(\frac{\left(\operatorname{Vol}\left(D_{t}\right)\right)}{\operatorname{Vol}\left(B_{t}^{b, 2}\right)}\right)^{\prime} \\
& +\int_{\partial D_{t}}<B^{P}(e, e), \frac{\nabla^{\perp} r}{\left\|\nabla^{P} r\right\|}>d \sigma_{t}
\end{aligned}
$$


The above equation is valid for all $t>R_{0}$, so, taking limits when $t \rightarrow \infty$, we can define

$$
\begin{aligned}
G_{b}(P) & :=\lim _{t \rightarrow \infty}\left(h_{b}(t) \operatorname{Vol}\left(B_{t}^{b, 2}\right)\left(\frac{\left(\operatorname{Vol}\left(D_{t}\right)\right)}{\operatorname{Vol}\left(B_{t}^{b, 2}\right)}\right)^{\prime}\right. \\
& \left.\left.+\int_{\partial D_{t}}<B^{P}(e, e), \frac{\nabla^{\perp} r}{\left\|\nabla^{P} r\right\|}>d \sigma_{t}\right)\right)
\end{aligned}
$$

Using equalities 4.5 , we have that

$$
G_{b}(P)=2 \pi \chi(P)+\frac{1}{2} \int_{D_{t}}\left\|B^{P}\right\|^{2} d \sigma-2 \pi \operatorname{Sup}_{t} \frac{\operatorname{Vol}\left(D_{t}\right)}{\operatorname{Vol}\left(B_{t}^{b, 2}\right)}<\infty
$$

and hence, $G_{b}(P)$ do not depends on the exhaustion $\left\{D_{t}\right\}_{t>0}$

Remark d. In view of equality (4.1) in Theorem 4.1 and equality (1.2) in the Euclidean setting, a question that naturally arises is if the quantity $G_{b}(P)$ is zero or at least set geometric conditions under which $G_{b}(P)$ is zero. One consideration in this direction is based on the assumption of a specific estimate on the curvature decay for cmi surfaces in the Hyperbolic space. With this specific curvature decay, we obtain a simplified expresion for $G_{b}(P)$.

Namely, given $P^{2}$ be a cmi surface in the real space form $\mathbb{K}^{n}(b),(b \leq 0)$, we say that the surface has spherical curvature decay in $\mathbb{K}^{n}(b)$ if and only if

$$
\sup _{x \in \partial D_{r}}\left\|B^{P}\right\|(x)<\frac{\epsilon(r)}{\operatorname{Vol}\left(S_{r}^{b, 1}\right)}
$$

when $r$ is big enough, where $\lim _{r \rightarrow \infty} \epsilon(r)=0, D_{r}$ is an extrinsic $r$ - ball in $P$, and $S_{r}^{b, 1}$ is the geodesic $r$-sphere in $\mathbb{K}^{n}(b)$.

With this definition in hand, it is easy to check that, if we consider an exhaustion of a cmi surface $P \subseteq \mathbb{H}^{n}(b)$ by extrinsic balls $\left\{D_{t_{i}}\right\}_{i=0}^{\infty}$ as in Corollary 3.3 we have:

$$
\lim _{i \rightarrow \infty} \frac{\operatorname{Vol}\left(B_{t_{i}}^{b, 2}\right)}{\operatorname{Vol}\left(D_{t_{i}}\right)} \int_{\partial D_{t_{i}}}<B^{P}(e, e), \frac{\nabla^{\perp} r}{\left\|\nabla^{P} r\right\|}>=0
$$

and hence we obtain (remind that $G_{b}(P)$ does not depend on the exhaustion):

$$
-\chi(P)=\frac{1}{4 \pi} \int_{P}\left\|B^{P}\right\|^{2}-\operatorname{Sup}_{t>0} \frac{\operatorname{Vol}\left(D_{t}\right)}{\operatorname{Vol}\left(B_{t}^{b, 2}\right)}-\lim _{i \rightarrow \infty}\left(h_{b}\left(t_{i}\right) \operatorname{Vol}\left(B_{t_{i}}^{b, 2}\right)\left(\frac{\left(\operatorname{Vol}\left(D_{t_{i}}\right)\right)}{\operatorname{Vol}\left(B_{t_{i}}^{b, 2}\right)}\right)^{\prime}\right)
$$

To proof the assertion 4.9, we use the fact that $P$ has spherical curvature decay. Hence, as

$$
\sup _{x \in \partial D_{r}}\left\|B^{P}\right\| \leq \frac{\epsilon(r)}{\operatorname{Vol}\left(S_{r}^{b, 1}\right)}
$$

for $\mathrm{r}$ big enough, we have, integrating around $\partial D_{t}$ for sufficiently large $t$ and using co-area formula:

$$
\frac{-\epsilon(t)\left(\operatorname{Vol}\left(D_{t}\right)\right)^{\prime}}{\operatorname{Vol}\left(S_{t}^{b, 1}\right)} \leq \int_{\partial D_{t}}<B^{P}(e, e), \frac{\nabla^{\perp} r}{\left\|\nabla^{P} r\right\|}>\leq \frac{\epsilon(t)\left(\operatorname{Vol}\left(D_{t}\right)\right)^{\prime}}{\operatorname{Vol}\left(S_{t}^{b, 1}\right)}
$$

Now, we consider the extrinsic exhaustion given by Corollary 3.3 and let $i \rightarrow \infty$ to have the proof, having into account that $\lim _{r \rightarrow \infty} \epsilon(r)=0$.

We note that Anderson obtained the estimate of the curvature decay of complete minimal surfaces in $\mathbb{R}^{n}$ with finite total curvature given by Proposition 2.2 in [1], so it is 
easy to see that all minimal surfaces in $\mathbb{R}^{n}$ with finite total scalar curvature have spherical curvature decay. In this case we have, on the other hand, the equality

$$
-\chi(P)=\frac{1}{4 \pi} \int_{P}\left\|B^{P}\right\|^{2}-\operatorname{Sup}_{t>0} \frac{\operatorname{Vol}\left(D_{t}\right)}{\operatorname{Vol}\left(B_{t}^{b, 2}\right)}
$$

However, when $P$ is a complete miminal surface immersed in $\mathbb{H}^{n}(b)$ with finite total extrinsic curvature, then $\left\|B^{P}\right\|(p)$ goes to zero when the extrinsic distance of $p$ to a fixed pole in $\mathbb{H}^{n}(b)$ goes to infinity (see [21]), but it was not given any explicit estimate of the curvature decay in this case.

To finish, we should remark that the complete and embedded spherical catenoids in $\mathbb{H}^{3}(-1)$, (see [17]), have finite total extrinsic curvature and spherical curvature decay, (see [24] and [14]).

\section{REFERENCES}

[1] M. T. Anderson, The compactification of a minimal submanifold in Euclidean space by the Gauss map , I.H.E.S. Preprint, 1984

[2] M. T. Anderson, Complete minimal varieties in Hyperbolic space, Invent. Math., 69, (1982), 477-494.

[3] S.S. Chern and R. Osserman Complete minimal surfaces in euclidean space (Academic Press, New York, 1984).

[4] S.S. Chern and R. Osserman, Complete minimal surface in $E^{n}$, J. d'Analyse Math. 19 (1967), 15-34.

[5] Chen Qing, On the area growth of minimal surfaces in $\mathbb{H}^{n}$, Geometriae Dedicata, 75 (1999), 263273.

[6] Chen Qing and Cheng Yi, Chern-osserman inequality for minimal surfaces in $\mathbb{H}^{n}$, Proc. Amer. Math Soc., Vol. 128, 8, (1999), 2445-2450.

[7] Chen Qing. On the volume growth and the topology of complete minimal submanifolds of a Euclidean space J. Math. Sci. Univ. Tokyo 2 (1995), 657-669.

[8] A. Esteve and V. Palmer Chern-Osserman Inequalities for surfaces in Cartan-Hadamard manifolds, Preprint, (2010)

[9] A. Esteve, V. Gimeno and V. Palmer Compactification of surfaces in Manifolds with a pole, Preprint , (2010)

[10] R. Greene and S. Wu Function theory on manifolds which posses a pole, Lecture Notes in Math.,699, (1979), Springer Verlag, Berlin.

[11] A. Huber, On subharmonic functions and differential geometry in the large, Comment. Math. Helv., 32 (1957), 13-72.

[12] A. Hurtado, S. Markvorsen and V. Palmer, Torsional rigidity of submanifolds with controlled geometry, Math. Ann. 344 (2009), 511-542.

[13] L.P. Jorge and W. H. Meeks, The topology of minimal surfaces of finite total Gaussian curvature, Topology, 122, (1983), 203-221.

[14] A. Kasue and K. Sugahara, Gap theorems for certain submanifolds of Euclidean spaces and Hyperbolic space forms, Osaka J. Math, 24, (1987), 679-704.

[15] M. Kokubu, M. Umehara, K. Yamada, Minimal surfaces that attain equality in the Chern-Osserman Inequality, Differential geometry and integrable systems, Tokyo 2000, Contemp. Math., 308, (2002), 223-228.

[16] J. Milnor Morse theory, Lecture Notes in Math.,699, (1979), Springer Verlag, Berlin.

[17] H. Mori, Minimal surfaces of revolution in $\mathrm{H}^{3}$ and their global stability, Indiana Univ. Math. Jour., 30, 5, (1981), 787-794.

[18] S. Markvorsen and V. Palmer, The relative volume growth of minimal submanifolds, Archiv der Mathematik, 79 (2002), 507-514.

[19] S. Markvorsen and V. Palmer, Extrinsic isoperimetric analysis on submanifolds with curvatures bounded from below, J. Geom. Anal. 20 (2010) 388-421.

[20] S. Muller and V. Sverak, On surfaces of finite total curvature, J. Differential Geometry, 42, 2, (1995), 229-257.

[21] G. De Oliveira, Compactification of minimal submanifolds of hyperbolic space, Comm. An. and Geom., 1 (1993), 1-29.

[22] V. Palmer, Isoperimetric Inequalities for extrinsic balls in minimal submanifolds and their applications, J. London Math. Soc. (2) 60 (1999), 607-616.

T. Sakai, Riemannian Geometry (Addison-Wesley, Reading, MA 1990). 
[26] K. Shiohama, Total curvature and minimal areas of complete open surfaces, Proc. Amer. Math. Soc.,, 94, num. 2, (1985), 310-316.

[27] B. White, Complete surfaces of finite total curvature, J. Diff. Geom., 26 (1987), 315-326.

Departament de Matemàtiques, Universitat Jaume I, Castelló, SPAin.

E-mail address: gimenov@guest.uji.es

Departament de Matemàtiques, Universitat Jaume I, Castelló, Spain.

E-mail address: palmeremat.uji.es 